\title{
Interrelationship between depression, anxiety, pain, and treatment adherence in hemophilia: results from a US cross-sectional survey
}

This article was published in the following Dove Press journal: Patient Preference and Adherence

\author{
Michelle L Witkop (D) \\ Angela Lambing $\mathbb{D}^{2}$ \\ Cynthia D Nichols ${ }^{3}$ \\ James E Munn ${ }^{4}$ \\ Terry L Anderson ${ }^{5}$ \\ Bartholomew J Tortella $\mathbb{D}^{5}$ \\ 'Nursing Department, Northern \\ Regional Bleeding Disorders Center, \\ Munson Medical Center, Traverse City, \\ MI, USA; ${ }^{2}$ Nursing Department, Henry \\ Ford Hemophilia and Thrombosis \\ Treatment Center, Detroit, MI, USA; \\ ${ }^{3}$ Nursing Department, Munson Medical \\ Center, Traverse City, MI, USA; \\ ${ }^{4}$ Hemophilia \& Coagulation Disorders \\ Program, University of Michigan \\ Hemophilia Treatment Center, Ann \\ Arbor, MI, USA; ${ }^{5}$ Medical Affairs, Pfizer \\ Inc, Collegeville, PA, USA
}

Purpose: Depression, anxiety, pain, and treatment adherence have reciprocal effects not characterized extensively in hemophilia. This study explored the relationships between depression, anxiety, chronic pain, and treatment adherence in adults with hemophilia.

Patients and methods: Adults with self-reported hemophilia A or B completed the crosssectional IMPACT QoL II survey. Depression (9-item Patient Health Questionnaire [PHQ-9]), anxiety (7-item Generalized Anxiety Disorder scale [GAD-7]), chronic pain (Faces Pain ScaleRevised [FPS-R]), social support (Duke UNC Functional Social Support questionnaire), level of pain control, clotting factor treatment adherence (VERITAS-Pro or -PRN), and previous depression/anxiety were analyzed.

Results: Among 200 participants (male, 77.3\%; female, 22.8\%), 54\% had PHQ-9 and 52\% had GAD-7 scores indicating moderate to severe depression or anxiety without diagnosis of either disorder. Participants with PHQ-9 scores $\geq 10$ (moderate to severe depression) were more likely to have lower treatment adherence than those with PHQ-9 scores $<10(P<0.05)$. Participants with PHQ-9 or GAD-7 scores $\geq 10$ were more likely to report uncontrolled pain and less social support versus PHQ-9 or GAD-7 scores $<10\left(\chi^{2} P<0.05\right)$. Significant correlations were found between PHQ-9 and GAD-7 $(P<0.0001)$, PHQ-9 and FPS-R $(P=0.0004)$, PHQ-9 and VERITAS $(P=0.01)$, GAD-7 and FPS-R $(P=0.02)$, and GAD-7 and VERITAS $(P=0.001)$.

Conclusion: Depression and anxiety are underdiagnosed in hemophilia. Depression is associated with anxiety, pain, and lower treatment adherence. While treatment providers play an important role in diagnosis, social workers may play a pivotal role in depression and anxiety screening. This study highlights the importance of regular screening and treatment for these disorders.

Keywords: blood coagulation disorders, depressive disorder, mental disorders, comorbidity, patient compliance, social support

\section{Plain language summary}

This study was designed to help researchers and patients understand relationships between symptoms of depression and/or anxiety, level of pain control, presence of a social support system (friends/family), and adherence to clotting factor treatment among adults with hemophilia A or B. Two hundred adults recruited from state/national hemophilia meetings were asked to complete an online survey describing their hemophilia-related symptoms and experiences. Most reported symptoms of depression and/or anxiety; more than half who reported moderate-to-severe symptoms of depression or anxiety were not diagnosed previously with the disorder. Participants with moderate-to-
Correspondence: Michelle LWitkop

National Hemophilia Foundation, 7 Penn Plaza, Suite 1204, New York, NY 1000I, USA

Tel + I $34754 \mid 2963$

$\mathrm{Fax}+$ I 2123283777

Email mwitkop@hemophilia.org 
severe symptoms of depression were more likely to have moderate-to-severe symptoms of anxiety and less likely to take their clotting factor according to the prescribed schedule versus those with no or mild symptoms. Participants with moderate-to-severe symptoms of depression or anxiety were more likely to report uncontrolled pain and less social support than those with mild or no symptoms. These results suggest that patients with hemophilia may have undiagnosed depression or anxiety and should be screened for these disorders so that treatment, if necessary, may be initiated. Treatment of depression or anxiety may improve adherence to clotting factor therapy and may improve the level of pain control in patients with hemophilia.

\section{Introduction}

Pain in chronic health conditions is often associated with depression. ${ }^{1,2}$ Pain, depression, and anxiety have complex, bidirectional, and often self-perpetuating interrelationships. Suboptimal coping strategies often affect relationships and reduce motivation to participate in activities that increase self-worth. This may worsen depression or anxiety, ${ }^{3}$ leading to a harmful cycle of depression and behavioral inertia. Few studies describe the prevalence or predictors of depression or anxiety among adult persons with hemophilia (PWH). Available evidence suggests that depression screening and care may improve overall health outcomes and treatment adherence in PWH. $^{4}$

We evaluated data from the Interrelationship Between Mood, Pain, Adherence to Clotting Factor Treatment, and Quality of Life (IMPACT-QoL II) survey to determine the prevalence of depression or anxiety among adults of both sexes diagnosed with hemophilia. We also explored relationships between depression, anxiety, chronic pain, perceived social support, and adherence to clotting factor treatment.

\section{Methods}

\section{Participants}

Study participants were recruited at 1 national bleeding disorder meeting, 2 inhibitor summits, and 2 large state meetings in 2013 and 2014 utilizing medical social workers (MSWs) as data managers owing to the sensitive mental health topic. Eligible individuals were men or women aged $\geq 18$ years; had self-reported diagnoses of hemophilia A or B; and could read, write, and speak English. The study aimed to enroll 150-200 participants.

\section{Ethics}

Investigators obtained prior authorization from relevant conference planning committees before recruitment. The study was approved by the institutional review board at Munson Medical Center (Traverse City, MI, USA), the primary investigator's institution when the study was conducted. Consent to participate was "click to consent" presented prior to any survey.

\section{Assessments}

The IMPACT QoL II, a cross-sectional, 139-item survey, comprises multiple validated patient assessment tools. ${ }^{5-13}$ Participants completed the IMPACT QoL II with on-site study computer tablets using SurveyMonkey ${ }^{\mathrm{TM}}$ (www.sur veymonkey.com), an online questionnaire program. Use of on-site computers prevented the collection of participants' IP addresses, safeguarding their privacy. Following the survey, participants received a \$20 Amazon gift card. Only specific variables from the original IMPACT QoL II survey were used in this analysis.

\section{Depression and anxiety}

The Patient Health Questionnaire 9-item depression module (PHQ-9) ${ }^{8}$ and the Generalized Anxiety Disorder 7-item scale $(\mathrm{GAD}-7)^{7}$ are validated measures of depression and anxiety that have been incorporated into numerous clinical practice guidelines, studies, and practice care settings; thus, they are used in the current analysis. ${ }^{14}$ The diagnostic validity of PHQ-9 in adults was established in studies involving $>6000$ patients in 8 primary care and 7 obstetrical clinics. ${ }^{15,16}$

The GAD-7 scale has strong criterion validity in the identification of cases of generalized anxiety disorder, which is one of the most common mental health disorders in outpatients. ${ }^{7}$ The scale also reflects severity in that GAD-7 scores increase with multiple domains of functional impairment and number of disability days. The use of the GAD-7 is important in a study focused on depression because, although many patients have both anxiety and depressive symptoms, previous studies confirmed and depression as distinct dimensions. As a limitation, the GAD-7 scale focuses only on generalized anxiety disorder.

In the current study, a score of $\geq 10$ on the PHQ- 9 or GAD-7 was designated as the criterion for moderate to severe depression or anxiety, respectively, based on the literature. $^{7,8,17}$ Trained MSWs were utilized as data managers in the recruitment phase. Prior to closing the survey 
and releasing the gift card, an MSW reviewed the PHQ-9 for any signs of suicidality. Resources were available for participants, if needed. None were utilized during the recruitment phase.

\section{Pain}

The Faces Pain Scale-Revised (FPS-R) is a self-reported assessment of acute pain felt during a bleeding event or chronic pain felt every day or almost every day, scored on a scale of $0-10$. The FPS-R shows a strong positive correlation with the visual analog scale for pain. The reliability and validity of the FPS-R has been established in pediatric subjects as young as 4 years old and in adults and has performed well with multiple racial and ethnic minorities. ${ }^{11,12}$ Participants were also asked about the level of control they had over their pain, using questions adapted from a nonvalidated tool from a previous study of acute and chronic pain in patients with bleeding disorders. ${ }^{18}$

\section{Adherence to clotting factor replacement therapy}

The Validated Hemophilia Regimen Treatment Adherence Scale-Prophylaxis (VERITAS-Pro) and VERITAS-OnDemand (VERITAS-PRN) were used to evaluate adherence to prescribed prophylactic and on-demand clotting factor treatment regimens, respectively. ${ }^{6}$ They are the only validated treatment adherence scales designed for use in PWH. Both instruments are 24-item questionnaires divided into six 4-item subscales that evaluate specific components of treatment adherence, as well as global adherence, to prescribed treatment regimens. Participants completed the VERITAS-Pro if they used their clotting factor as routine prophylaxis or before physical activity. Participants completed the VERITAS-PRN if they used their clotting factor only for treating bleeding events. Both the VERITAS-Pro and VERITAS-PRN can be completed by the patient or by an observer. Responses are chosen from a 5-point Likert scale ranging from "always" to "never"; an answer of "always" reflects the best possible adherence for some items and the worst possible adherence for others (items are scored in different directions, depending on whether their wording reflects adherence or non-adherence). Subscale scores ranged from 4 points (most adherent) to 20 points (least adherent), and total scores ranged from 24 (most adherent) to 120 (least adherent). Adherence was measured by VERITAS scores and dichotomized as lower (ie, scores in the highest quartile) and higher (ie, scores in the lowest quartile).

\section{Social support}

The Duke-UNC Functional Social Support Questionnaire (DUFSS) is a validated measure of social support. ${ }^{10}$ The scale contains 8 items; 5 items assess social support, and 3 items assess affective support. Each response is scored on a scale of 1-5. The average score increases as the level of social support increased.

\section{Statistical analyses}

All analyses were conducted using Stata v15.0 (StataCorp, LLC; College Station, TX, USA). For descriptive statistics, categorical variables were described with percentages and frequency counts. Pearson chi-square was used to compare groups when all cells had $\mathrm{n}>10$. The 2-sided Fisher's exact test was used to compare groups when any cell had $\mathrm{n}<10 .{ }^{19}$ The level of statistical significance was set at $P<0.05$.

For statistical modeling on noncategorical variables, separate Generalized Linear Models were built for depression (PHQ-9 score) and anxiety (GAD-7 score) to assess the relationship of each to the level of pain, perceived social support (DUFSS score), and adherence to the participant's prescribed clotting factor regimen. Diagnostic techniques were used for each statistical approach to ensure proper discrimination, fit, and specification of each model, and to verify the validity of underlying statistical assumptions. Based upon the result of the models, individual comparisons between variables with absolute value $\mathrm{z}$-scores $>1$ were performed using 2-tailed $t$ tests for unequal variances. Correlations were performed using Spearman's rho with Bonferroni correction for multiple comparisons. ${ }^{19}$

\section{Results}

The study population comprised a convenience sample of 200 adults (male, $\mathrm{n}=146$ [77.3\%], female $\mathrm{n}=43$ [22.8\%]) aged $\geq 18$ years with self-reported hemophilia A $(80.5 \%$ [161/200]) or B (19.5\% [39/200]). Demographic and clinical characteristics of study participants are outlined in Table 1. Participants were recruited in 2013 and 2014 at national or state conferences for PWH in the United States. Each participant completed all surveys that were appropriate for that individual. For example, based upon their reported treatment regimen, participants were assigned to 
Table I Demographic and clinical characteristics of study participants

\begin{tabular}{|l|l|}
\hline Parameter & Patients (N=200) \\
\hline Age, mean (range), years ${ }^{\mathrm{a}}$ & $36.5(18-86)$ \\
\hline Sex, $\mathrm{n}(\%)^{\mathrm{a}}$ & \\
Male & $146(77)$ \\
Female & $43(23)$ \\
\hline Race, $\mathrm{n}(\%)^{\mathrm{a}}$ & \\
White & $128(68)$ \\
Black & $27(14)$ \\
Asian & $3(2)$ \\
Native American or Alaskan native & $2(\mathrm{I})$ \\
Native Hawaiian or other Pacific islander & $2(\mathrm{I})$ \\
Other & $27(14)$ \\
\hline Hemophilia type, $\mathrm{n}(\%)^{\mathrm{b}}$ & \\
A & $16 \mathrm{I}(8 \mathrm{I})$ \\
B & $39(20)$ \\
\hline Hemophilia severity, $\mathrm{n}(\%)$ & \\
Mild & $48(24)$ \\
Moderate & $16(8)$ \\
Severe & $136(68)$ \\
\hline Type of treatment, $\mathrm{n}(\%)^{\mathrm{b}}$ & \\
On-demand & $8 \mathrm{I}(4 \mathrm{I})$ \\
Before activity & $1 \mathrm{I}(6)$ \\
Prophylaxis & $108(54)$ \\
\hline Inhibitor status, $\mathrm{n}(\%)^{\mathrm{b}}$ & $79(40)$ \\
Yes & $12 \mathrm{I}(6 \mathrm{I})$ \\
No & \\
\hline
\end{tabular}

Notes: ${ }^{a} \mathrm{n}=189$. Sex and race for II patients are unknown. ${ }^{\mathrm{b}}$ Percentages may not add to $100 \%$ because of rounding.

complete either the VERITAS Pro (prophylactic treatment regimens) or PRN (episodic treatment regimens).

\section{PHQ-9 and prior diagnosis of depression}

Of 186 participants who identified symptoms of depression (ie, score $>0), 28 \%(\mathrm{n}=52)$ reported moderate-tosevere symptoms of depression, and 50\% (26/52) of those reported using on-demand therapy. However, of these 52 patients, more than half reported no history of a diagnosis for depression (Figure 1A).

\section{GAD-7 and prior diagnosis of anxiety}

Of 184 participants who identified symptoms of anxiety (ie, score $>0), 13 \%(n=23)$ reported moderate-to-severe symptoms of anxiety, and 74\% (17/23) of those reported using on-demand therapy. More than half of participants reported no prior anxiety diagnosis in both the moderate- to-severe anxiety group and in the mild or no anxiety group although those with moderate to severe anxiety on the GAD-7 were more likely to have been diagnosed $\left(\chi^{2}=9.90 ; P<0.01\right)$ (Figure 1B). Of the 81 participants who reported using on-demand clotting factor therapy, 76 (94\%) had at least one symptom of anxiety on the GAD-7.

\section{GAD-7, PHQ-9, and social support}

In total, 193 of 200 participants provided data for the GAD-7, PHQ-9, and DUFSS subanalyses. Of these, $13 \%(n=25)$ reported moderate-to-severe symptoms of anxiety and $22 \%$ $(\mathrm{n}=42)$ reported moderate-to-severe symptoms of depression. Participants with scores $\geq 10$ on either the GAD-7 or PHQ-9 had significantly lower scores (less perceived social support) on the DUFSS than patients with scores $<10$ on those scales (GAD-7: 3.5 vs 4.3, $\mathrm{t}=3.121, P=0.004$; PHQ-9: 3.4 vs 4.4; $\mathrm{t}=5.691, P \leq 0.0001$ ) (Figure 2).

\section{Treatment adherence}

All participants completed either VERITAS measure. A quartile analysis indicated that VERITAS scores of $<39$ and $>57$ corresponded to the 25 th and 75 th percentiles, respectively, which were the lowest and highest quartiles. In total, 95 patients had VERITAS scores in the 25th or 75th percentile. While there was not a linear relationship, participants with VERITAS scores in the 75th quartile (lower treatment adherence; $n=49$ ) were more likely to have PHQ- $9 \geq 10$ than participants in the 25th quartile (higher treatment adherence; $\mathrm{n}=46$ ) (Fisher's exact test, $P=0.024$ ), whether on prophylaxis or on-demand therapy (Figure $3 \mathrm{~A}$ ). Adherence in participants with GAD-7 scores $\geq 10$ was numerically lower versus participants with GAD-7 scores $<10$; however, the difference was not significant (Fisher's exact test, $P=0.149$; Figure 3B).

In total, $41 \%$ of participants in the PHQ-9 analysis and $40 \%$ of participants in the GAD-7 analysis reported using an on-demand clotting factor treatment regimen. When stratified according to the clotting factor treatment regimen, those who reported using clotting treatment on demand had significantly higher mean PHQ-9 or GAD-7 scores (greater severity of depression or anxiety) compared with those who reported using clotting treatment before physical activity or prophylactically (PHQ-9: 7.43 vs $5.57, \mathrm{t}=2.212, P=0.029$; GAD-7: 6.53 vs $4.81, \mathrm{t}=2.497$, $P=0.007)$.

\section{Level of pain control}

Among participants who provided data on the level of pain control, 30.6\% (59/193) reported uncontrolled pain. 


\section{A}

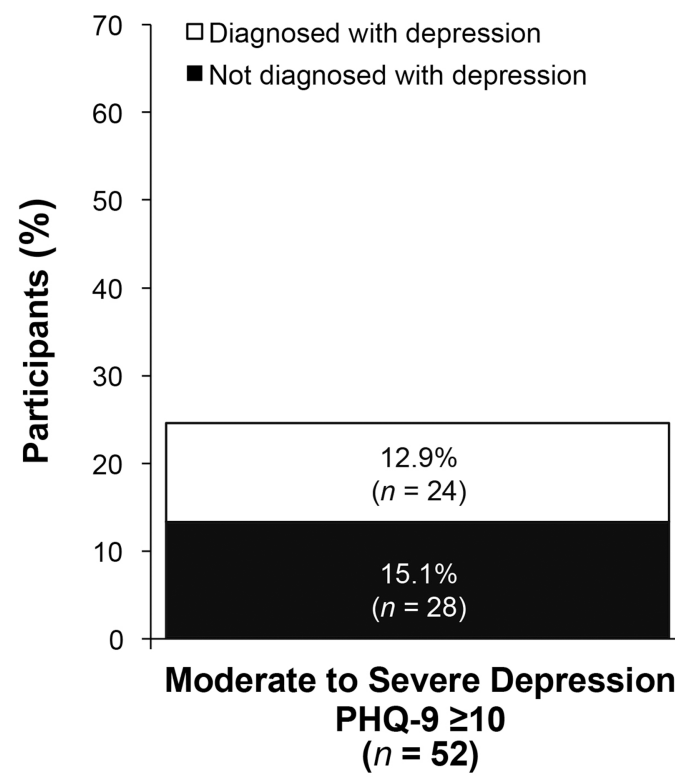

B

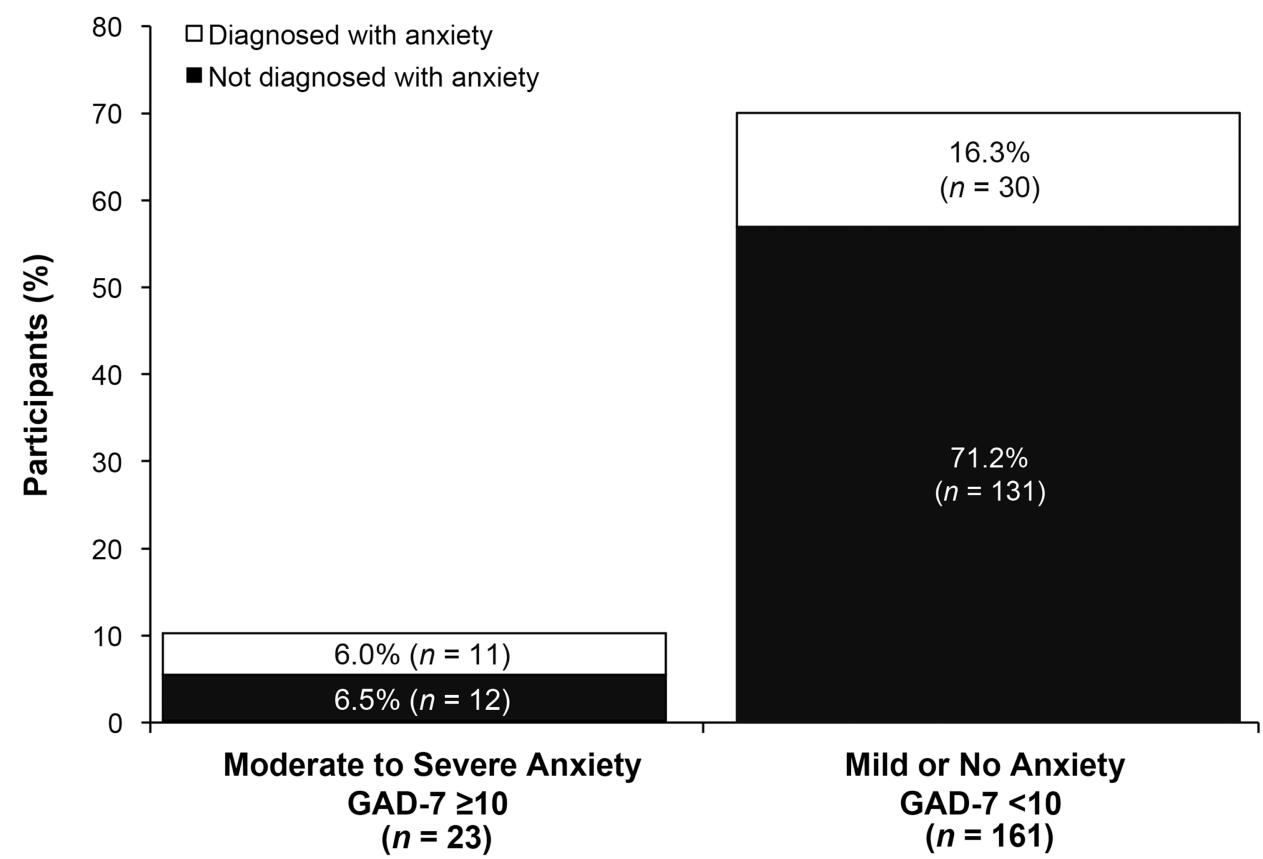

Figure I Prevalence of diagnosed depression, by PHQ-9 score ${ }^{\mathrm{a}}(\mathbf{A})$ and of diagnosed anxiety, by GAD-7 score ${ }^{\mathrm{b}}(\mathbf{B})$. Percentages were based on $\mathrm{n}=186$ and $\mathrm{n}=184$, respectively. $P \leq 0.001$ and $P<0.01$, based on $\chi^{2}$ analysis, respectively.

Notes: ${ }^{\text {aPHQ}}-9$ score $\geq 10$ was designated as the criterion for moderate to severe depression, and PHQ-9 score $<10$ was the criterion for mild or no depression, based on the literature. ${ }^{8}$ bAD-7 score $\geq 10$ was designated as the criterion for moderate-to-severe anxiety, and GAD-7 score $<10$ was the criterion for mild or no anxiety, based on the literature. ${ }^{7}$ Abbreviations: GAD-7, 7-item Generalized Anxiety Disorder scale; PHQ-9, 9-item Patient Health Questionnaire depression scale.

A greater proportion of patients had uncontrolled pain and scores $\geq 10$ on the PHQ-9 or GAD-7 than uncontrolled pain and scores $<10 \quad\left(\chi^{2}=44.948\right.$, $P<0.0001$ for the PHQ-9; $X^{2}=6.214, P=0.013$ for the GAD-7) (Figure 4). The percentage of participants with uncontrolled pain was similar between those who reported using clotting treatment on demand and those who reported using clotting treatment before physical activity or prophylactically $(27.5 \%$ [22/80] vs $32.7 \%$ [37/113], $\mathrm{X}^{2}=0.601, P=0.436$ ). 


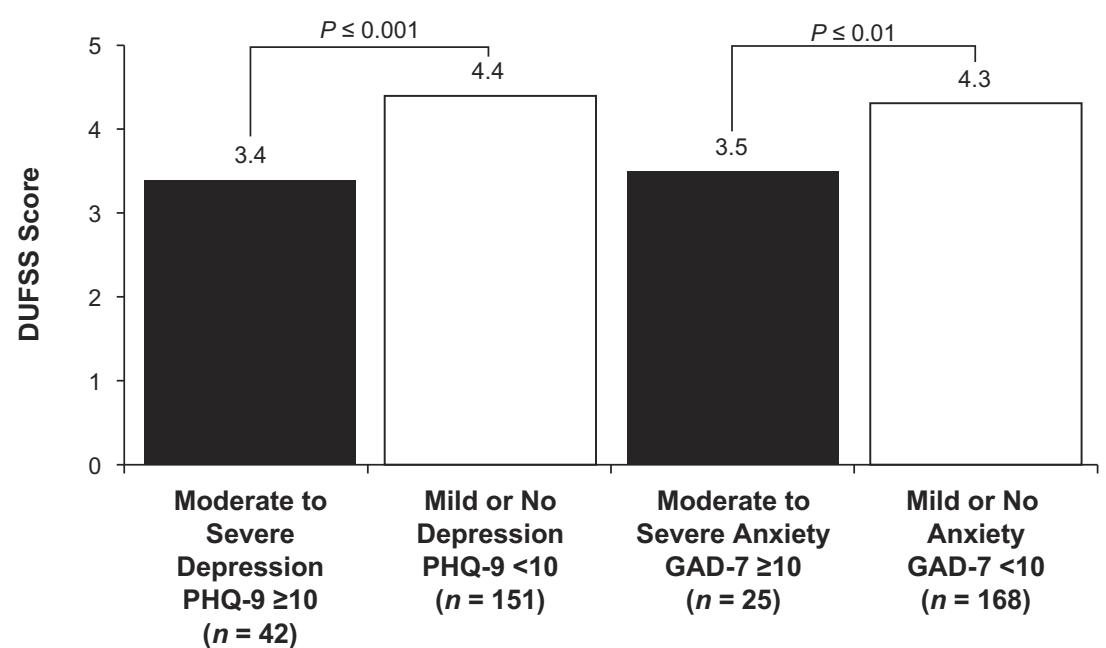

Figure 2 Perceived social support, measured on the DUFSS, stratified by severity of depression and anxiety. The scale contains 8 items; 5 items assess social support, and 3 items assess affective support. Each response is scored on a scale of $1-5$. The average score increases as the level of social support increases.

Abbreviations: DUFSS, Duke-UNC Functional Social Support Questionnaire; GAD-7, 7-item Generalized Anxiety Disorder scale; PHQ-9, 9-item Patient Health Questionnaire depression scale.

Correlations between anxiety, depression, chronic pain, and adherence were statistically significant, and positive correlations were found between PHQ-9 and GAD-7 scores $(\mathrm{Rho}=0.767, P<0.0001$ ), indicating that as PHQ-9 scores increased, GAD-7 scores also increased (ie, as depression severity increased, anxiety severity also increased). A significant correlation with medium effect size was observed between PHQ-9 and FPS-R (chronic pain) scores (Rho $=0.308, P=0.0004)$ and between GAD-7 and FPS-R scores ( $\mathrm{Rho}=0.216, P=0.02)$, indicating that as depression or anxiety severity increased, chronic pain intensity also increased. Lastly, statistically significant correlations were observed between PHQ-9 and VERITAS (adherence) scores (Rho $=0.181, P=0.01$ ) and between GAD-7 and VERITAS scores (Rho $=0.228, P=0.001$ ), indicating that as depression and anxiety severity increased, treatment adherence decreased (Table 2).

\section{Discussion}

In this study of adult PWH, depression, anxiety, pain, and low adherence to clotting factor treatment were found to be interrelated. Each of these issues is known to exist in PWH. Among women with hemophilia $\mathrm{B}$ in the Bridging Hemophilia B Experiences, Results and Opportunities into Solutions (B-HERO-S) study, $43 \%$ reported anxiety and $42 \%$ reported depression. ${ }^{20}$ Overall, $89 \%$ of adult PWH report experiencing pain that interfered with activities during the previous month. ${ }^{21}$ Chronic pain is also common in hemophilia. In a study of patients with hemophilia B, $40 \%$ of those aged $>45$ years reported chronic pain. Arthritis is a common comorbidity in these patients; $55 \%$ of the patients with moderate hemophilia B and $59 \%$ of the patients with severe hemophilia B report having arthritis. ${ }^{20}$ In general, PWH have a higher risk of disability and pain with increasing age. $^{21}$ Among PWH with severe disease, the majority (66\%) adhered to clotting factor treatment $<70 \%$ of the time. $^{22}$

In total, $28 \%$ of the participants had PHQ-9 scores indicating moderate-to-severe depression compared with a $6.7 \%$ prevalence in the general adult population (females, $8.5 \%$ vs males, $4.8 \%$ ). ${ }^{23}$ In comparison, $13 \%$ of the participants had GAD-7 scores indicating moderate-to-severe anxiety compared with an estimated $19 \%$ of the adults who had any anxiety disorder in the last year (females, $23.4 \%$ vs males, $14.3 \%$ ). ${ }^{24}$ Further, more than half of the participants in our study who scored in the moderate-to-severe range on validated screening tests for depression or anxiety had never received a diagnosis for either of these disorders. Collectively, these results support the need for more widespread screening for depression and anxiety in PWH. This study also found that depression is associated with lower adherence to clotting factor treatment in adult PWH. Failure to diagnose depression may be a barrier to optimizing treatment of both disorders. The availability of an MSW as a member of the hemophilia treatment center core team offers the opportunity for depression and anxiety screening at every comprehensive visit and possibly at every visit when deemed appropriate. 

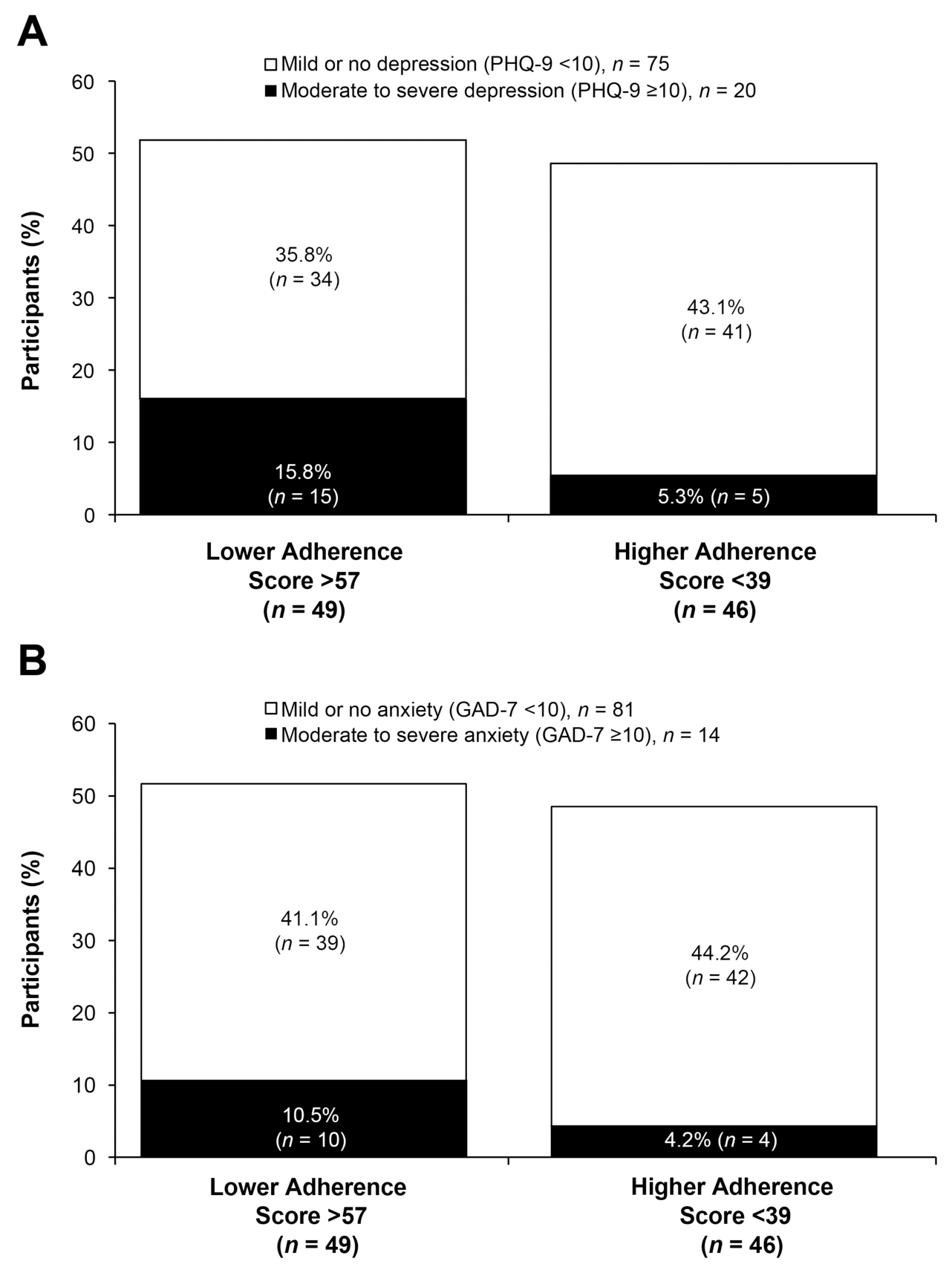

Figure 3 Prevalence of treatment adherence, stratified by presence of moderate-to-severe (A) depression $\left(P<0.05, \chi^{2}\right.$ analysis) or (B) anxiety ( $P=N S, \chi^{2}$ analysis).apercentages were based on $\mathrm{n}=95$.

Notes: ${ }^{\mathrm{a} B a s e d}$ on the literature, PHQ-9 score $\geq 10$ and GAD-7 score $\geq 10$ were designated as the criteria for moderate-to-severe depression and anxiety, respectively; PHQ-9 score $<10$ and GAD-7 score $<10$ were designated as the criteria for mild or no depression and anxiety. ${ }^{7,8}$

Abbreviation: PHQ-9, 9-item Patient Health Questionnaire depression scale.

In this study, $41 \%$ of the participants in the PHQ-9 analysis and $40 \%$ of the participants in the GAD-7 analysis reported using an on-demand clotting factor treatment regimen. This is approximately twice as high as the proportion reported in the B-HERO-S study $(20 \%){ }^{20}$ As the study population for B-HERO-S is limited to patients with hemophilia $\mathrm{B}$, limited data are available for comparison. $\mathrm{PWH}$ with depression scores in the moderate-to- severe range were more likely to have lower adherence to clotting factor treatment, regardless of regimen (ie, prophylactic, before physical activity, or on demand) than high adherence ( $15.8 \%$ vs $5.3 \%$, respectively; $P<0.05)$. Another study in $\mathrm{PWH}$ showed that a history of depression predicted a 7-point higher score on the VERITAS-Pro or VERITAS-PRN (ie, lower adherence). ${ }^{25}$ 


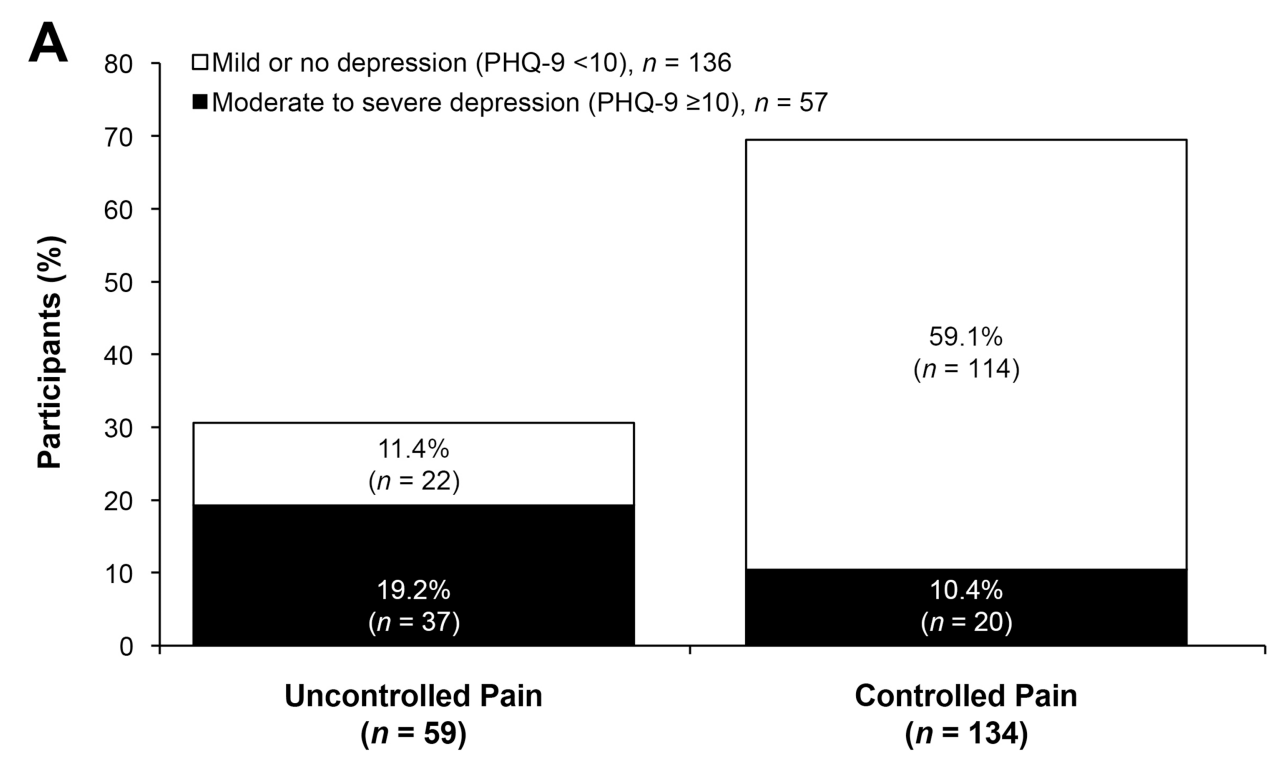

B

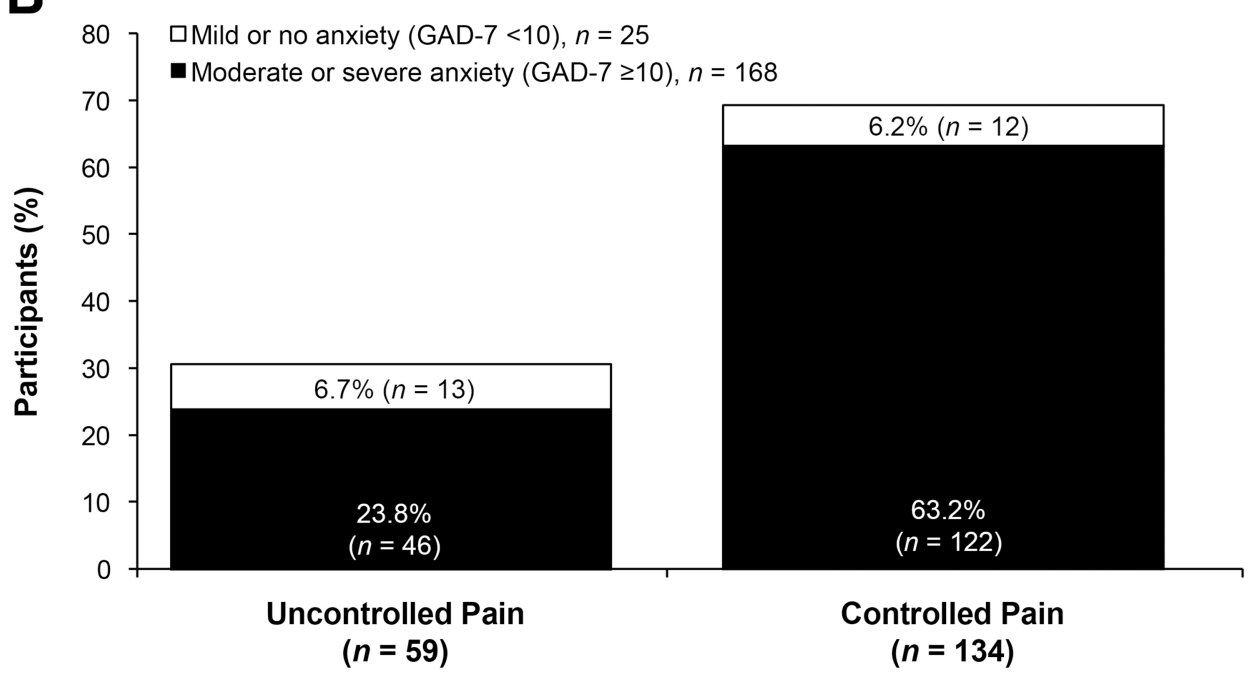

Figure 4 Prevalence of participants' level of pain control, stratified by presence of moderate-to-severe $(\mathbf{A})$ depression $\left(P \leq 0.00\right.$ I, $\chi^{2}$ analysis $)$ or $(\mathbf{B})$ anxiety $\left(P<0.05, \chi^{2}\right.$

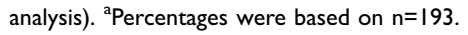

Notes: ${ }^{\text {BB }}$ ased on the literature, PHQ-9 score $\geq 10$ and GAD-7 score $\geq 10$ were designated as the criteria for moderate-to-severe depression and anxiety, respectively; PHQ-9 score $<10$ and GAD-7 score $<10$ were designated as the criteria for mild or no depression and anxiety, respectively. ${ }^{7,8}$

Abbreviations: GAD-7, 7-item Generalized Anxiety Disorder scale; PHQ-9, 9-item Patient Health Questionnaire depression scale.

Table 2 Correlation matrix of level of depression, anxiety, treatment adherence, and pain ${ }^{\mathrm{a}}$

\begin{tabular}{|l|l|l|l|l|}
\hline Parameter & PHQ-9 & GAD-7 & VERITAS $^{\mathbf{b}}$ & FPS-R $^{-1}$ \\
\hline PHQ-9 & - & - & - & - \\
\hline GAD-7 & $0.77(P<0.001)$ & - & - & - \\
\hline VERITAS & $\mathrm{N}=197$ & $0.23(P=0.001)$ & - & - \\
\hline FPS-R & $0.18(P=0.01)$ & $\mathrm{N}=198$ & & \\
& $\mathrm{~N}=197$ & $0.22(P=0.02)$ & $-0.004(P=0.961)$ \\
$\mathrm{N}=127$ & $\mathrm{~N}=127$ \\
\hline
\end{tabular}

Notes: ${ }^{a}$ Correlation parameters (Spearman's rho and corresponding P-value) between variables are shown. ${ }^{b}$ VERITAS-Pro or VERITAS-PRN. ${ }^{\mathrm{C}}$ Differences in patient numbers are because those who reported that they did not have chronic pain were not asked to the complete the FPS-R.

Abbreviations: FPS-R, Faces Pain Scale-Revised; GAD-7, 7-item Generalized Anxiety Disorder scale; NA, not applicable; NS, not significant; PHQ-9, 9-item Patient Health Questionnaire depression scale; VERITAS, Validated Hemophilia Regimen Treatment Adherence Scale. 
In the current study, PWH with moderate or severe symptoms of depression or anxiety were more likely to report uncontrolled pain than PWH with mild or no symptoms of depression or anxiety. The mechanism by which pain potentially worsens both depression and anxiety may involve the sharing of neural pathways. ${ }^{26}$ The presence of comorbid depression in patients with chronic pain is problematic because it may be associated with an increased risk of opioid misuse, ${ }^{27}$ a problem that has reached epidemic proportions in the US.

Studies have supported bidirectionality between pain, anxiety, depression, and treatment adherence. For example, chronic illness and depression have reciprocal effects in which the presence of one can exacerbate or increase the risk of the other. ${ }^{4}$ It has been proposed that chronic illness itself does not increase the risk of depression or anxiety, but the pain associated with chronic disorders increases the risk of these disorders. ${ }^{3}$ In the current study, PWH with moderate or severe symptoms of depression or anxiety were more likely to report uncontrolled pain than PWH with less severe symptoms of depression or anxiety. Patients with pain and depression or anxiety may have reduced motivation and decreased ability to engage in behaviors that improve pain management, including adherence to a clotting factor treatment regimen or use of a clotting factor to treat pain (presumed bleeding event) arising from hemophilia-associated arthritis. Overall, the combination of pain and depression predicts worse clinical outcomes compared with either condition alone. ${ }^{26}$ Efforts should be directed toward the diagnosis and treatment of depression and anxiety in PWH, with the goal of improving both adherence to treatment and quality of life. Additional studies are needed to confirm whether treatment adherence and pain management are improved when comorbid depression and anxiety are effectively treated.

These results underscore the potential value of social service and support in $\mathrm{PWH}$, which cannot be overemphasized. PWH lacking social service and support (from family, friends, or their community) are 5 times more likely to report depressive symptoms than PWH who felt supported ( 0.20 odds ratio; $95 \%$ confidence interval, 0.04 $0.96 ; P<0.05){ }^{4}$ The roles defined by each hemophilia treatment center for social workers vary widely, and no data to describe role responsibility are available. Therefore, depression and anxiety screening should be conducted as part of the normal comprehensive clinic assessment by hemophilia treatment center team members with referral to outside resources when necessary. Increased support to social work colleagues through increased time and funding of positions would aid in appropriate screening. In this study, participants with higher depression and anxiety scores reported having lower levels of social support. This relationship is also likely to be bidirectional and self-perpetuating. In the BHERO-S study of patients with hemophilia B, $10 \%$ of adults surveyed had visited a social worker and only $1 \%$ of those with moderate disease severity had a social worker on their care team. ${ }^{20}$ Given the mutually reciprocal effects of depression and anxiety on pain and treatment adherence and the large percentage of PWH who previously had not been diagnosed with depression in this study $(72 \%)$, it is important to practice routine screening for depression and anxiety disorders during patient visits to hemophilia treatment centers. Once diagnosed, prompt initiation of an effective treatment plan may encourage healthy coping skills, increase treatment adherence, and improve clinical outcomes in $\mathrm{PWH}$.

We recognize the limitations of a study such as this. Because this was a cross-sectional observational study, causality could not be determined. The use of self-reported patient information and the intention to protect patient privacy prevented study personnel from objectively verifying the data collected in the survey. Although parts of the survey used validated instruments, such as the PHQ-9, GAD-7, VERITAS-Pro, and VERITAS-PRN, these are not the equivalent of clinical diagnostic tools. Other parts of the survey used for this study were not previously validated. In correlation analyses, some variables failed to reach statistical significance, but this may have been the result of smaller sample sizes for those particular correlations. Additionally, generalizability of the results may be limited by recruitment bias, given that study participants were recruited through hemophilia advocacy groups and may not be representative of all adult PWH. Finally, while these data were collected from 2013 to 2014, several other publications have utilized these data. We recognize there have been many changes in the bleeding disorder landscape since that time. Overall, however, this information is relevant, new, and germane to the current environment, although this could be considered a limitation of the study.

\section{Conclusion}

This study sheds light on potentially modifiable, bidirectional variables that can affect the course of disease in PWH. Many PWH may have undiagnosed depression or anxiety. On-demand therapy regimens are associated with increased depression and anxiety scores. Depression, 
anxiety, uncontrolled pain, insufficient social support, and suboptimal treatment adherence are likely interdependent. Our data support this construct and demonstrate that depression, anxiety, and uncontrolled pain are associated with reduced adherence to treatment. A multidisciplinary team within the hemophilia treatment center that facilitates assessment and treatment of patients' depression, anxiety, and social support needs may have a positive impact on improving clinical outcomes and treatment adherence. Further research on the topic of depression and anxiety in the bleeding disorder community in general, and specifically in those with hemophilia, is recommended, with a focus on how these issues affect adherence to medications, treatment plans, and attendance at the treatment center.

\section{Abbreviations}

DUFSS, Duke-UNC functional social support questionnaire; FPS-R, Faces Pain Scale-Revised; GAD-7, 7-item Generalized Anxiety Disorder scale; IMPACT-QoL II, interrelationship between mood, pain, adherence to clotting factor treatment, and quality of life; NA, not applicable; NS, not significant; PHQ-9, 9-item Patient Health Questionnaire depression scale; PWH, person with hemophilia; VERITAS, Validated Hemophilia Regimen Treatment Adherence Scale.

\section{Acknowledgment}

The authors thank John M. McLaughlin, PhD, of Pfizer Inc, for his participation in the development of the original survey and for previous statistical analysis, and Christi Humphrey, MSW, for her review of this manuscript and expert opinion. The authors thank all of the survey participants for their time in contributing to this study. Jessica D. Herr, PharmD, and Barbara Zeman, PhD, of Peloton Advantage, LLC, an OPEN Health company, provided medical writing and editorial support, which were funded by Pfizer Inc.

\section{Author contributions}

All authors contributed to data analysis, drafting and revising the article, gave final approval of the version to be published, and agree to be accountable for all aspects of the work.

\section{Disclosure}

This study was sponsored by Pfizer Inc. MLW reports grants from Pfizer, Inc, during the conduct of the study. JEM has served on the advisory boards of Takeda, NovoNordisk, Genentech, CSL Behring, Octapharma, Shire/Baxalta and as Consultant for Bayer outside the submitted work. AL is currently employed by Bayer HealthCare. TLA is an employee of
Pfizer Inc and may own stock/options in the company. BJT was an employee of Pfizer Inc at the time of manuscript preparation. CDN has no conflicts to disclose.

\section{References}

1. Fuller-Thomson E, Shaked Y. Factors associated with depression and suicidal ideation among individuals with arthritis or rheumatism: findings from a representative community survey. Arthritis Rheum. 2009;61(7):944-950. doi:10.1002/art.24615

2. Balliet WE, Edwards-Hampton S, Borckardt JJ, et al. Depressive symptoms, pain, and quality of life among patients with nonalcohol-related chronic pancreatitis. Pain Res Treat. 2012;2012:978646.

3. Gerrits MM, van Oppen P, Leone SS, van Marwijk HW, van der Horst HE, Penninx BW. Pain, not chronic disease, is associated with the recurrence of depressive and anxiety disorders. BMC Psychiatry. 2014;14:187. doi:10.1186/1471-244X-14-187

4. Iannone M, Pennick L, Tom A, et al. Prevalence of depression in adults with haemophilia. Haemophilia. 2012;18(6):868-874. doi:10.1111/ j.1365-2516.2012.02863.x

5. Duncan NA, Kronenberger WG, Roberson CP, Shapiro AD. VERITASPRN: a new measure of adherence to episodic treatment regimens in haemophilia. Haemophilia. 2010;16(1):47-53. doi:10.1111/j.13652516.2009.02094.x

6. Duncan N, Kronenberger W, Roberson C, Shapiro A. VERITASPro: a new measure of adherence to prophylactic regimens in haemophilia. Haemophilia. 2010;16(2):247-255. doi:10.1111/ j.1365-2516.2009.02129.x

7. Spitzer RL, Kroenke K, Williams JB, Lowe B. A brief measure for assessing generalized anxiety disorder: the GAD-7. Arch Intern Med. 2006;166(10):1092-1097. doi:10.1001/archinte.166.10.1092

8. Kroenke K, Spitzer RL, Williams JB. The PHQ-9: validity of a brief depression severity measure. J Gen Intern Med. 2001;16(9):606-613. doi: $10.1046 / j .1525-1497.2001 .016009606 . x$

9. Radloff LS. The CES-D scale: a self-report depression scale for research in the general population. Appl Psychol Meas. 1977;1 (3):385-401. doi:10.1177/014662167700100306

10. Broadhead WE, Gehlbach SH, de Gruy FV, Kaplan BH. The DukeUNC functional social support questionnaire. Measurement of social support in family medicine patients. Med Care. 1988;26(7):709-723. doi:10.1097/00005650-198807000-00006

11. Hicks CL, von Baeyer CL, Spafford PA, van Korlaar I, Goodenough B. The Faces Pain Scale-Revised: toward a common metric in pediatric pain measurement. Pain. 2001;93(2):173-183. doi:10.1016/ s0304-3959(01)00314-1

12. Ware LJ, Epps CD, Herr K, Packard A. Evaluation of the revised faces pain scale, verbal descriptor scale, numeric rating scale, and iowa pain thermometer in older minority adults. Pain Manag Nurs. 2006;7(3):117-125. doi:10.1016/j.pmn.2006.06.005

13. Ware JE Jr., Sherbourne CD. The MOS 36-item short-form health survey (SF-36). I. Conceptual framework and item selection. Med Care. 1992;30(6):473-483.

14. Kroenke K, Wu J, Yu Z, et al. Patient health questionnaire anxiety and depression scale: initial validation in three clinical trials. Psychosom Med. 2016;78(6):716-727. doi:10.1097/PSY.0000000000000322

15. Spitzer RL, Kroenke K, Williams JB. Validation and utility of a selfreport version of PRIME-MD: the PHQ primary care study. Primary care evaluation of mental disorders. Patient health questionnaire. JAMA. 1999;282(18):1737-1744. doi:10.1001/jama.282.18.1737

16. Spitzer RL, Williams JB, Kroenke K, Hornyak R, McMurray J. Validity and utility of the PRIME-MD patient health questionnaire in assessment of 3000 obstetric-gynecologic patients: the PRIME-MD patient health questionnaire obstetrics-gynecology study. Am J Obstet Gynecol. 2000;183 (3):759-769. doi:10.1067/mob.2000.106580 
17. Manea L, Gilbody S, McMillan D. Optimal cut-off score for diagnosing depression with the patient health questionnaire (PHQ-9): a meta-analysis. CMAJ. 2012;184(3):E191. doi:10.1503/ cmaj. 110829

18. Witkop M, Lambing A, Kachalsky E, Divine G, Rushlow D, Dinnen J. Assessment of acute and persistent pain management in patients with haemophilia. Haemophilia. 2011;17(4):612-619. doi:10.1111/j.13652516.2010.02479.x

19. Stata statistical software: release 15. StataCorp; 2017. Available from: https://www.stata.com/. Accessed July 16, 2019.

20. Buckner TW, Witkop M, Guelcher C, et al. Management of US men, women, and children with hemophilia and methods and demographics of the bridging hemophilia B experiences, results and opportunities into solutions (B-HERO-S) study. Eur J Haematol. 2017;98 (Suppl 86):5-17. doi:10.1111/ejh.12854

21. Forsyth AL, Witkop M, Lambing A, et al. Associations of quality of life, pain, and self-reported arthritis with age, employment, bleed rate, and utilization of hemophilia treatment center and health care provider services: results in adults with hemophilia in the HERO study. Pat Prefer Adher. 2015;9:1549-1560. doi:10.2147/PPA.S87659
22. Armstrong EP, Malone DC, Krishnan S, Wessler MJ. Adherence to clotting factors among persons with hemophilia A or B. Hematology. 2015;20(3):148-153. doi:10.1179/1607845414Y.0000000176

23. Mental health information: major depression. Bethesda, MD: National Institute of Mental Health; 2017. Available from: https://www.nimh.nih. gov/health/statistics/major-depression.shtml. Accessed July 16, 2019.

24. Mental health information: any axiety disorder. Bethesda, MD: National Institute of Mental Health; 2017. Available from: https://www.nimh.nih. gov/health/statistics/any-anxiety-disorder.shtml. Accessed July 16, 2019.

25. Tran DQ, Barry V, Antun A, Ribeiro M, Stein S, Kempton CL. Physician trust and depression influence adherence to factor replacement: a single-centre cross-sectional study. Haemophilia. 2017;23 (1):98-104. doi:10.1111/hae.13078

26. Bair MJ, Robinson RL, Katon W, Kroenke K. Depression and pain comorbidity: a literature review. Arch Intern Med. 2003;163 (20):2433-2445. doi:10.1001/archinte.163.20.2433

27. Finan PH, Carroll CP, Moscou-Jackson G, et al. Daily opioid use fluctuates as a function of pain, catastrophizing, and affect in patients with sickle cell disease: an electronic daily diary analysis. J Pain. 2018;19(1):45-56. doi:10.1016/j.jpain.2017.08.010
Patient Preference and Adherence

\section{Publish your work in this journal}

Patient Preference and Adherence is an international, peer-reviewed, open access journal that focusing on the growing importance of patient preference and adherence throughout the therapeutic continuum. Patient satisfaction, acceptability, quality of life, compliance, persistence and their role in developing new therapeutic modalities and compounds to optimize clinical outcomes for existing disease

\section{Dovepress}

states are major areas of interest for the journal. This journal has been accepted for indexing on PubMed Central. The manuscript management system is completely online and includes a very quick and fair peer-review system, which is all easy to use. Visit http:// www.dovepress.com/testimonials.php to read real quotes from published authors. 